\title{
Capitate-Hamate Joint
}

National Cancer Institute

\section{Source}

National Cancer Institute. Capitate-Hamate Joint. NCI Thesaurus. Code C139188.

The articulation of the capitate and hamate bones in the wrist. 